III. Aus der medicinischen Universitätsklinik in Bonn.

\section{Ueber die Behandlung des Empyems mittels der Heberdrainage.}

\section{Von Dr. K. Bohland, Privatdocenten und Assistenten der} medicinischen Klinik.

Wenn auch heute bei der Behandlung des Empyems nur noch die Rippenresection und die Büla a 'sche Heberdrainage als die einzig berechtigten Methoden in Frage kommen, so hat doch die Heberdrainage noch mit mannigfachen, wie uns scheint, unbegründeten Vorurtheilen zu kämpfen.

Herr Professor Schultze hat auf der hiesigen Klinik das $B$ ülau'sche Verfahren eingeführt, und wenn auch bisher nur 6 Fälle zur Beobachtung kamen, so scheint es doch angemessen bei dem jetzigen Stande der Dinge, auch über eine so geringe Anzahl zu berichten, wobei bemerkt werden muss, dass im folgenden über alle derartigen Fälle der Klinik Rechenschaft abgelegt wird.

I. Empyem nach einer Stichverletzung. Anamnese: Patient R., 33 Jahre alt, will früher nie krank gewesen sein. 3 Wochen vor seiner Aufnahme ist er im Streite mit einem Messer in die rechte Brusthălfte gestochen worden. Unmittelbar danach befiel ihn heftige Athemnoth, er wurde tief cyanotisch (Pneumothorax?). Die A themnoth verringerte sich allmählich, es trat Fieber ein und Stechen in der rechten Seite. Es bestand kein Husten, kein Nachtschweiss, Appetit war ziemlich gut.

Status praesens: Patient ist ein kräftig gebauter Mensch. Im zweiten rechten Intercostalraum besteht ein Abscess, aus dessen Fistel sich eine grössere Menge eines rahmigen Eiters entleert; eine Communication mit dem Pleuraraume lässt sich nicht nachweisen. Rechts beginnt eine Dămpfung in der Mammillarlinie an der 6. Rippe, in der mittleren Axillarlinie gleichfalls an der 6. Rippe und hinten an der Wirbelsäule an der 7. Rippe. Die rechte Spitze ist $2 \mathrm{~cm}$ hoch, der Schall über ihr kürzer als über der linken Spitze, die $3 \mathrm{~cm}$ hoch ist. Das Athmungsgeräusch, dem nirgends Rhonchi beigemengt sind, ist auf der ganzen rechten Seite sehr schwach; der Pectoralfremitus ist rechts hinten unten aufgehoben. An der linken Lunge ist ausser einer stärkeren Ueberlagerung des Herzens durch dieselbe nichts abnormes zu finden. Das Herz ist gesund, keine deutliche Verdrängung desselben. Im Harn kein Eiweiss. Die Probepunction in der rechten Seite, vorgenommen im 6. Intercostalraum, ergiebt Eiter. Das Empyem wächst in den nächsten 10 Tagen noch langsam, so dass vorn die Dämpfung von der 4. Rippe, in der Seite von der 5. Rippe und hinten an der 6. Rippe beginnt. Während dieser Zeit fiebert Patient des Abends măssig, nur einmal 39,3 , sonst meist nur 38,0 .

Am 28. November wird die Drainage nach Bülau vorgenommen, und ca. $1000 \mathrm{ccm}$ eines dicken, rahmigen Eiters werden entleert; täglich wird 3 mal mit warmer Salicylsåurelösung ausgespült. Die Temperatur sinkt nun auch abends auf die Norm, nur an 3 Tagen wird abends noch $38,0-38,5$ notirt gefunden (an diesen Tagen war das Spülen mittags unterlassen

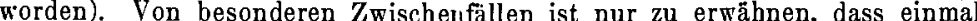
am Ende der ersten Woche der Drainage bei sehr heftigen Huṣtenstössen der Katheter herausgestossen worden war; es entstand danach kein ausgedehnterer Pneumothorax, und der Katheter konnte ohne weiteres wieder in die Pleuraböhle eingeschoben werden. Die Eitersecretion wurde allmählicb geringer; es flossen beim Spülen immer kleinere Mengen der Spülflüssigkeit in den Pleuraraum ein. Nachdem am 15. Januar seit einigen Tagen stets nur geringfügige Mengen von Fibringerinnseln uud kein Eiter mehr entleert war, und nur wenige Cubikcentimeter Flüssigkeit beim Spülen einliefen, wurde der Katheter, der inzwischen mehr nach aussen gezogen war, ganz entfernt. Die Fistel war nach 10 Tagen völlig geschlossen, und nun entleerte sich auch aus der Fistel im 2. Intercostalraum, die bis dahin noch täglich grössere Mengen eines rahmigen Eiters secernirt hatte, kein Secret mehr.

Der Lungenbefund bei der Entlassung war folgender: Die rechte Thoraxhälfte bleibt beim Athmen kaum noch zurück. Vorn in der Mammillarlinie rechts Dämpfung an der 6. Rippe, in der Seite an der 8. Rippe, hinten unten an der 11. Rippe, während links hinten unten die Lungengrenze an der 12. Rippe steht. Das Athmungsgeräusch ist oben rechts normal, in den unteren Partieen gleichfalls normal, nur etwas schwächer als links.

Wir haben also in diesem Falle eine fast völlige Restitutio ad integrum durch das Bülau'sche Verfahren erzielt; die Behandlungsdauer betrug 50 Tage.

II. Empyem nach Influenzapneumonie. Anamnese: Patient K., 19 Jahre alt, stammt aus ganz gesunder Familie. Weihnachten 1889 war er an Influenza erkrankt; am 3. Januar 1890 bekam er Schüttelfrost, Fieber und Auswurf; vom Arzt wird eine Pneumonie diagnosticirt. Seit dieser Zeit klagt Patient bis heute, 11. Mai 1890, über Schmerzen in der rechten Seite, Athemnoth, geringen Appetit.

Status praesens: Patient ist sehr abgemagert, die Schleimhäute blass, die Respiration ist beschleunigt, und zwar wird nur die linke Thoraxhälfte bewegt. Rechte Thoraxpartie vorgewölbt, Intercostalräume daselbst verstrichen. Unter der Clavicula besteht rechts absolute Dämpfung bis zur Leber hinunter, ebenso in der rechten Seite absolute Dämpfung, hinten rechts bis zur 3. Rippe tympanitischer Schall, von da ab bis unten hin Dämpfung. Die Grenzen der linken Lunge deutlich erweitert. Links vorn und hinten verschärftes Inspirium, rechts unten Athmungsgerăusch fehlend, oben rechts leise zu hören, dasselbe hat bronchialen Charakter. Der Pectoralfremitus ist rechts hinten unten völlig aufgehoben. Das Herz ist verdrängt, der Spitzenstoss in der vorderen linken Axillarlinie. Die Leber überragt $3 \mathrm{~cm}$ den Rippenbogenrand. Es besteht kein Fieber, im Harn kein Albu- min. Um der Gefahr eines Collapses beim raschen Ablass des Eiters zu entgehen, wird zunächst die einfache Punction vorgenommen.

Am 12. Mai werden durch Punction $1500 \mathrm{ccm}$ eines dicken, rahmigen Eiters entleert, wonach der Spitzenstoss mehr nach rechts rückt.

Am 16. Mai wird die Aspirationsdrainage angelegt; durch dieselbe fiessen nun nochmals 2 l Eiter ab. In den folgenden Tagen werden noch weitere Eitermengen durch den Katheter entleert, im ganzen 6 l. Während bei den täglichen Spülungen anfangs $400 \mathrm{ccm}$ Salicylsâurelösung einfliessen, fasst allmählich der Pleuraraum nur noch bis zu $22 \mathrm{ccm}$ Flüssigkeit. Am 22. Juli wird der Katheter aus der Pleurahöhle entfernt; die Fistol heilt schon nach 9 Tagen völlig zu.

Die Behandlung nahm hier 68 Tage in Anspruch und verlief ohne jeden $\mathrm{Zwischenfall,} \mathrm{Patient} \mathrm{sieht} \mathrm{blühend} \mathrm{aus;} \mathrm{die} \mathrm{Lungen-}$ grenzen sind rechts völlig normal, die Lunge athmet gut, ein Resultat, das um so auffallender ist, als es sich hier doch um ein jedenfalls mehrere Monate bestehendes Empyem handelt.

IIl. Jauchiges Empyem. Anamnese: Patient Th., 52 Jahre alt, bat vor 24 Jahren eine Lungenentzündung durchgemacht. Seit ca. 6 Wochen hat er Schmerzen in der rechten Seite, Athemnoth, Husten und schleimigeitrigen Auswurf; seit 14 Tagen bestehen Kopfschmerzen, leichter Schwindel, allgemeine Mattigkeit und zeitweilig plötzlich eintretendes Frieren. Seit vielen Jahren hat Patient Zittern der Hände, Alkoholmissbrauch wird zu. gegeben.

Status praesens: Patient ist ein kräftig gebauter Mensch, liegt mit stark geröthetem Gesicht zu Bett, fiebert leicht

Rechts beginnt am oberen Rande der 5. Rippe in der Mammillarlinie bsolute Dämpfung, in der Axillarlinie an der 5. Rippe, hinten an der 6. Rippe. Pectoralfremitus fehlt rechts hinten unten, das Athmungsgeräusch ist unten hinten deutlich bronchial und ziemlich laut. Es bestehen keine Verdrängungserscheinungen von Seiten des Herzens und der Leber. Am Berzen nichts abnormes, Puls langsam, regelmässig, sehr gespannt. Im Sputum, das schleimig-eitrig und foetid ist, sind keine Tuberkelbacillen zu finden, im Harn ist keiu Albumin.

Die Probepunction, im 9 I. C. Raum hinten rechts vorgenommen, ergiebt jauchig riechende trübe Flïssigkeit. Patient ist nachts unruhig; trotzdem er Chloral abends erhält, verlässt er das Bett. Am 22. November wird die Bülau'sche Drainage angelegt, und in 3 Stunden fliessen $2^{1 / 2}$ Liter einer scheusslich stinkenden Jauche $a b$; am anderen Tage findet man in dem Spülwasser 7ahlreiche lange Fibrinfetzen, in denen keine geschichteten Membranen und keine Echinococcenstücken zu finden sind. Während die Temperatur vor der Drainage abends meist bis 39,0 stieg, fiel sie nach derselben sofort zur Norm, und nur einmal finden wir abends 38,6 verzeichnet. Es werden Spülungen mit Salicylsäure oder mit Pyoktaninlösungen 1:2000 vorgenommen.

In den ersten Wochen war der Auswurf sehr gering, am 17. Januar werden plötzlich copiöse Mengen (200 ccm) eines sehr fötiden Auswurfes entleert. Hinten rechts unten finden wir an der Wirbelsäule lantes Bronchialathmen. Das Allgemeinbefinden des in der Nacht durch einen Wärter behüteten Patienten ist ein ausgezeichnetes.

In der zweiten Woche der Drainage glitt der Katheter bei Hustenstössen aus der Pleurahöhle heraus, derselbe liess sich leicht wieder einführen, kein Pneumothorax war nachzuweisen. Sonst hat eine Unterbrechang der Hebervorrichtung nicht stattgefunden. Am 29. Januar kann die Drainage völlig entfernt werden; die Menge des Sputums ist immer noch ziemlich reichlich; dasselbe ist rein eitrig und nicht mehr fötid. Die Percussion ergiebt die untere Lungengrenze rechts vorne an der 7. Rippe, ebenso in der Seite und hinteu leichte Dämpfung von der 8. Rippe ab; das Athmungsgeräusch ist deutlich vesiculär, auch hinten, und nicht mehr bronchial.

Die Menge des eitrigen Sputums beträgt ca. $10 \mathrm{ccm}$; in demselben sind keine Truberkelbacillen nachweisbar.

Die Heilung des unter so schwierigen Verhältnissen bei einem unruhigen Potator drainirten Empyems nahm 68 Tage in Anspruch und führte, abgesehen von einer geringen Schrumpfung der Lunge hinten unten, zu einem sehr guten Resultat.

IV. Jauchiges Empyem (nach Durchbruch eines Leberechinoc occus in die Pleurahöhle). Anamnese: Pationtin H., 56 Jahre alt, war angeblich stets gesund, bis sie im December 1889 Influenza bekam. Seit dieser Zeit magerte sie ab, wurde hinfällig und matt, da bei starker Anorexie sich andauernde Diarrhoeen einstellten.

Status praesens am 25. Februar 1890: Patientin sieht cacisektisch aus; es besteht Dämpfung rechts hinten von der 4. Rippe, in der Axillarlinie von der 6. Rippe, in der Mammillarlinie von der 4. Rippe ab. Athmungsgeräusch dort abge

Die Lebergrenze ist palpabel, reicht in der Mittellinie bis unterhalb des Nabels, geht von da leicht im Bogen nach rechts und links bis zur Mammillarlinie. Die Oberfläche der Leber ist gleichmässig glatt, die Consistenz derselben ist hart. Das Abdomen ist aufgetrieben. Es besteht starkes Oedem an den Beinen und am Rücken. Im Harn, täglich $150-500 \mathrm{ccm}$, ist ziemlich viel Albumin vorhanden, es finden sich graunlirte Harncylinder. Patientin fiebert abends bis 38,80 , morgens nur bis 38,20 . Diarrhoeen bestehen auch hier noch fort, $2-3$ Stühle am Tage.

In den nächsten Nächten häufig asthmatische Anfälle (Collapse?), desam 18. März Punction, und dabei werden $1800 \mathrm{ccm}$ dicker jauchiger Flüssigkeit entleert. Danach hebt sich das Allgemeinbefinden, die Harnmengen steigen auf $2000 \mathrm{ccm}$ und enthalten nur wenig Eiweiss. Die Oedeme nehmen rasch ab.

Am 25. Närz wird die Heberdrainage angelegt; in dem jauchigen Eiter werden Membranen von Echinococcusblasen und Haken gefunden; mit der Gmelin'schen Reaction wird in dem Eiter Gallenfarbstoff nachgewiesen.

Auch in der Folge wird immer noch grünliche, übel riechende Flüssig- 
keit entleert. Patientin erholt sich von Tag zu Tag, das Eiweiss verschwindet vôllig aus dem Harn. Am 1. Mai wird die Drainage entfernt. Die Lungengrenzen werden nun rechts unten normal gefunden, ebenso das Athmungsgeräusch. Die Leber ist unverändert geblieben wie früher.

Bei dieser Patientin lehnte der Chirurg die Ausführung der Rippenresection ab, da er zur Zeit wegen der Schwäche und Albuminurie, die ja vielleicht der Ausdruck einer beginnenden Amyloidentartung gewesen ist, die Patientin nicht für geeignet hielt für eine derartige Operation. Die Heilung des Empyems erfolgte in einem Zeitraume von 36 Tagen, Patientin ist heute noch blühend und anscheinend völlig gesund.

V. Pyopneumothorax, Tuberculose der Lungen. Anamnese: Patient R., Bergmann, 33 Jahre alt, ist bis zum 5. September 1889 anscheinend völlig gesund gewesen. An diesem Tage bekam er plötzlich, wie er sagt „die Brustkrankheit", nämlich plötzlich Athemnoth, Fieber und Schmerzen in der linken. Seite. Früher will Patient, der aus ganz gesunder Familie stammt, zeitweise an Nachtschweissen gelitten haben. Status praesens am 15. November 1889: Patient ist ziemlich kräftig gebaut, aber stark abgemagert und sehr anämisch. Der Puls ist klein und frequent, es besteht Dyspnoe, die Muskeln am Halse wie Scaleni und Sternocleidomastoidei sind in Action bei den Athembewegungen. Die linke Seite bleibt beim Athmen fast völlig liegen. Linke Spitze ist nur $1 \mathrm{~cm}$ hoch, und der Schall über ibr kürzer als rechts. Unter der Clavicula links tympanitische Dämpfung, die an der vierten Rippe in absolute Dämpfung übergeht. In der Seite links gleichfalls absolute Dämpfung. Das Herz ist verlagert nach rechts, so dass die Herzdämpfung genau in der rechten Mammillarlinie beginnt; die Leberdämpfung ist nach unten vergrössert, so dass sie in der Mammillarlinie den Rippenbogenrand um $4^{1 / 2} \mathrm{~cm}$ übersebreitet. Links vorn oben hört man amphorisches Athmungsgeräusch, ferner exquisites Stäbchenklirren. Man kann deutlich das Schüttelgerãusch selbst auf einige Entfernung hin erzeugen. Beim Aufsetzen des Patienten verschwindet vorn links oben der tympanitische Klang, und man findet schon von der Clavicula an absolute Dämpfung. Auf der rechten Lunge, deren Grenzen nach unten erweitert sind, hört man überall verschärftes In- und Exspirium, aber nirgends Rhonchi.

Das Sputum ist schleímig-eitrig, enthält ziemlich viel Luft, Tuberkelbacillen werden nicht in ihm gefunden.

Patient fiebert besonders des Abends bis 38,5. Im Harn, aueh spăterhin zeitweise, Spuren von Albumin.

Am 18. November wurde die Drainage nach Bülau angelegt; es werden bis zum nächsten Tage $4850 \mathrm{ccm}$ nicht riechenden Eiters abgelassen, danach bessert sich die Athemnoth, das Herz kehrt in seine normale Lage zurück, Leberdämpfung überragt nur $1 \mathrm{~cm}$ den Rippenbogenrand, es tritt vorn links überall tief tympanitischer Schall an Stelle des gedämpften; Athmungsgeräusch ist vorn links nicht zu hören, die Temperatur sinkt zur Norm. In der Folge verstopft sich bäufiger die Canüle und dann entsteht öfter Fieber bis 39,6 , auch obne díese Ursache ist öfters Fieber vorhanden, und es lässt sich dasselbe durch Eingiessen von Jodoformolemulsion nicht deutlich beeinflussen. Allmählich aber verringert sich die Eitersecretion, man hört vorn links vesiculäres Athmungsgeräusch, während in der Seite deutlicher tympanitischer Schall ist. Bei einem heftigen Hustenanfall wird der $\mathrm{Ka}-$ theter herausgeschleudert, die Fistel schliesst sich leicht. Nach ca. 3 Wochen öffnet sich dieselbe wieder, es strömt Eiter in grösserer Menge aus ihr beraus.

Herz und Leber sind abermals verdrängt, und am 20. Januar 1890 wird abermals die Drainage nach Bülau angelegt und nun $1^{1 / 2} \mathrm{I}$ jauchigen Eiters entleert. Patient entleert jetzt Kohlensputum, in dem nun auch Tuberkelbacillen aufgefunden werden. Weiterbin verkleinerte sich allmählieh die Pleurahöhle, es floss beim Spülen weniger Flüssigkeit wie früher ein, das Athmungsgeräusch war vorn in grösserer Ausdehnung vesiculär, in der Seite und hinten dagegen abgeschwächt; nirgends amphorisches Athmen zu hören, hinten unten links begann die absolute Dämpfung an der 10. Rippe. Patient füblt sich meist leidlich wohl, steht am Tage einige Stunden auf, nachdem für diese Zeit an Stelle des Hebers ein einfaches Receptaculum gesetzt worden war.

Im Juli 1890 fiel Patient, nachdem er schon seit dem 7. April fast vëllig fieberfrei gewesen, beim Benutzen des Stechbeckens plötzlich um und verstarb wenige Minuten darauf.

Sectionsbericht (Prof. Köster): Lungen: Rechte Lunge etwas eingesunken, im rechten Pleuraraume eine grössere Quantităt leicht getrübter Flüssigkeit. Rechte Lunge nach hinten an der Basis mebrfach verwachsen. Das Sternum ist links fest mit dem Mediastinalgewebe verwachsen. Linke Pleura ist total in eine dicke Schwarte verwandelt, der ziemlich grosse freie Raum ist mit trüber dicklicher Flüssigkeit erfüllt.

Die rechte Lunge ist gross, in ihren vorderen Partieen noch lufthaltig, knisternd; in ihr sind einige knotige Verdichtungen; die Pleura stark. pigmentirt, an der Spitze schwarz pigmentirte eingezogene Narben. Die Schnitffäche der Lungen ist intensiv schwarz pigmentirt, so dass sich ein schwarzer Brei abstreifen lässt, ungefärbte Stellen nur in geringen Spuren; die indurirten Knoten lassen sich von dem nicht indurirten Gewebe nur durch das Gefühl unterscheiden. Im oberen Lappen mehrere kleine Knoten, an der Basis desselben eine alte Schwarte. Die Lymphdrüsen von derselben schwarzen Verdichtung.

Linkerseits von der Spitze bis nahe an die Basis findet sich ein Sack, aussen von der sehr verdickten Pleura costalis begrenzt; ob die innere Wand Pleura pulmonalis oder grob netzförmige Züge blossgelegten Lungengewebes, lässt sich nicht entscheiden.

Lungengewebe liegt bier in Inseln frei vor, vollständig "schwarz, an der Spitze sehr starke Verdichtungen. Lunge ist comprimirt, in der Grösse von 2 Händen, vollständig luftleer und schwarz, in ihr schlaffere und der- bere Stellen. Auf Druck auf die Lungen entleeren sich aus den Bronchien schleimige Massen mit schwarzen Zügen. Ulceration, Verkäsung oder der Tuberculose Verdächtiges ist in der Lunge nach der makroskopischen Betrachtung nicht vorhanden. An der Basis ist zwischen Lunge und Pleura diaphragmatica ein kleiner Spalt, in ihm breiiges Naterial (Käse?).

Herz: Klappen intact, Muskulatur dünn, blass, schlaff, keine Herderkrankungen.

In der linken Niere geht von einem weissen Fleck der Oberfläche ein weisser Zug bis zur Spitze eines Markkegels, hier viele Knötchen.

Rechte Niere ohne Veränderung. In der Leber, sowohl auf der Serosa als auf der Schnittfläche, weisse Knötchen.

In der Mitte des Ileum auf der Serosa eine Gruppe grauer Knötchen, innen ein markstückgrosses Ulous mit gewulsteten Rändern und Knötchen auf dem Grunde. Auf der Bauhin'schen Klappe abermals kleinere Ulcerationen. Die Rippen links sind von der dritten ab stark verdickt, springen leistenförmig vor.

Vl. Jauchiger Pyopneumothorax nach Typhus. Anamnese: Patient 0., 22 Jahre, will früher nie krank gewesen sein. In der Mitte des December 1890 erkrankte er an einem Typhus, an welchem er 5 Wochen zu Bett lag. Unmittelbar bieran ansehliessend traten Stiche in der linken Seite auf, auf's neue Fiebererscheinungen (Frösteln und Hitze); zugleich aber stellte sich Husten und Auswurf von sehr foetidem Sputum ein, das Sputum wurde nicht mundvollweise, sondern ständig in kleineren Mengen ausgehustet; es bestand weiter starke Dyspnoe.

Status am 24. Februar 1891: Patient ist ausserst anaemisch, die Athmung oberflächlich, sehr frequent, ebenso der Puls (144 in der Minufe). Auf der rechten Lunge ist nichts abnormes durch die Percussion und Auscultation zu finden. Die linke Spitze ist ehenso laut und hoch wie die rechte. Das Herz ist bis zur rechten Sternallinie verschoben; links vorn Dämpfung von der 3 . Rippe, an der linken Seite, wo die Percussion schmerzhaft ist, fast absolute Dämpfung. Links vorn obej schwaches Athmungsgeräusch, nach schwächer ist dasselbe in der Seite; hinten, wo eine absolute Uämpfing in der Yitte der Scapula beginnt, ist schwaches Bronchialathmen Lörbar. Stäbchenklirren ist nicht zu hören, wohl aber deutliches Schüttelgeräusch: ebenso ist die Verschieblichkeit der Flüssigkeit in der Pleurahöhle evident.

Patient fiebert bis 38,2 abends, im foetiden Sputum keine Tuberkelbacillen; durch die Probepunction in der linken Seite erhält man jauchige Flüssigkeit.

Am 26. Februar wird die Bülau'sche Drainage angelegt im 7. I. C. Raum in der linken Seite, nachdem mebrere Probepunctiouen im 5. und 6. I. C. Raum keinen Eiter ergeben hatten; es fliesst aus dem Katheter, der schwer einzusehieben war (dicke Schwarten) zunächst kein Eiter aus, wobl aber kann man $150 \mathrm{ccm}$ Salicylsäurelősung in die Höhle einlaufen lassen; mehr einzuführen verbieten die Klagen des Patienten über die nun sofort eintretende Athemnoth. Am 27. Februar fliessen plötzlich als dem Katheter $1_{1 / 2}^{1 / 2}$ Liter einer furchtbar stinkenden Flüssigkeit $a b$, die zahlreiche Streptococcen enthält; die Temperatur, die abends 38,9 gewesen war, fiel nun des Morgens auf 37,1. In der Umgebung der einen Probepunctionsstelle, die mit Eiterabfuhr im 7. I. C. Raum gemacht war, entsteht eine mãchtige Infiltration und Röthung: beim Einschnitt entleeren sich grosse Mengen jauchigen Eiters, und man gelangt von dem Einschnitt unmittelbar in die Pleurahöhle. Die Temperatur steigt wieder in die Höhe auf 39,2 .

Patient stirbt am 1. März abend̦ plötzlich im Collapse. Bis dahin war tändig Eiter aus dem Drainrohr abgeflossen.

Sectionsbericht (Prof. Köster): In der Gegend des 5. und 6. 1. C. Rauınes links um zwei Fistelöffnungen schwielige Verdichtungen des Zellgewebes, starkdurchfeuchtet, mit einigen Haemorrhagieen durchsetzt.

Linke Lunge in ganzer Ausdehnung, die rechte vielfach bandförmig erwachsen.

Rechte Pleurahöhle vorn ohne Flüssigkeit. Rechte Lunge sebr gross, leicht. Pleura glänzend, Verdichtungen nicht durchzufühlen. Auf der Schnittfläche guter Blut- und Luftgehalt, leichtes Oedem in den abhängigen Partieen. Verdiehtungen sind nicht zu erkennen.

Auf der linken Seite ist der Magen durch das Netz verklebt. Nach deren Lösung sieht man eine Oeffnung im $Z$ werchfell mit etwas schmutzig aufgeworfenem Rande. In dem aufgeklebten Netzstück schmutzige Verfärbung. Milz und Leber frei. Beim Ablösen der sehr festen, schwieligen Schwarte der linken Lunge kommt aus dem Raume zwischen Pleura costalis und Thoraxwand ein stark stinkender Brei heraus. Die Pleura costalis reisst vielfach ein, bildet die äussere Wand einer Höhle. Nach Eröffnung des grossen Sackes enthält derselbe theilweise festes schmutzig braungraues lappiges Gewebe. Die Lappen gehen nach unten in stark verdickte feste weissliche Pleura pulmonalis über. Der obere Lappen ist comprimirt uneben. Auf dem Durchschnitt lässt sich nicht mit Bestimmtheit sagen, ob die schmutzig graue Höble von der Pleura begrenzt ist oder von neugebildetem Gewebe. Eine ganze Reibe von Bronchien geht direkt an die auskleidende Membran; jedoch nur von einem Bronchus kann man bestimmt sagen, dass er durch einen Schlitz mit der Pleurahöhle in Communication steht, wăhrend der Bronchus selbst unversehrt bis zur Peripherie geht. In (ler Lunge selbst sind weder Verdichtungen noch Herderkrankungen, noch narbige Stränge vorhanden, mit Ausnahme der Basis, wo feste Verwachsungen existiren.

Herzbeutel blank; Muskulatur des Herzens kräftig, fleckig injicirt, aber ohne Herderkrankung.

Nieren zeigen nichts abnormes. Milz sehr gross.

Im Iloum vor der Klappe an Stelle der Peyer'schen Plaques, kleine graue Flecke, narbenähnlich. Einzelne lassen einen Defect erkennen mit grauen Răndern; die Erscheinung nimmt zu bis zur Klappe. Hier geschwellte Follikel. Im Dickdarm nichts abnormes. 
Zn der Ansführung des Bülan'schen Verfahrens branchen wir, da wir nns im wesentlichen genan an die Vorschriften des Erfinders der Methode gehalten haben, nur wenige Worte zn bemerken. Die in die Brnsthöhle eingeführten Nélaton'schen Katheter, an denen wir bald oben noch seitliche Löcher anbrachten, oder die wir anch oben an den Angen einfach abschnitten, haben wir danernd liegen lassen nnd sie niemals während der Behandlnng freiwillig entfernt. Nnr zweimal ist es nns passirt, dass bei heftigen Hnstenstössen die Katheter heransgeschlendert wnrden, nnd zwar geschah dies einmal Ende der ersten nnd einmal Ende der zweiten Woche.

Das Wiedereinführen des Katheters gelang beide male leicht, nnd kein ansgedehnterer Pnenmothorax war dann nachznweisen. Es scheint nus diese Thatsache dafür zn sprechen, dass anch bei Anwendnng des Bülan'schen Verfahrens die beiden Plenrablätter bei der Entfaltnng der Lnngen mit einander verkleben; dem entsprechend fanden wir anch bei Untersnchnng der geheilten Fälle, dass beim Athmen eine sehr ansgedehnte Verschiebnng der beiden Plenrablätter nicht stattfand.

Ansspülnngen wnrden von nns täglich, meist 3 mal gemacht und dazn warme Salicylsänrelösnngen oder anch Pyoktaninlösnngen (1: 2000) benntzt, ohne jedoch von letzteren einen besonderen Erfolg gesehen zn haben. Bülan räth nnn in seiner letzten Pnblication zn einem hänfigeren Wechsel der Katheter nnd hält die wiederholten Ansspülnngen für schädlich, vielleicht mag nnser Verfahren die Heilnngsdaner etwas verlängert haben.

Die Gefahr des Verstopfens der Drainageröhren halten wir nicht für sehr gross, obgleich wir öfters grössere Gerinnsel durch das Glasrohr geben sahen und anch das Sistiren des Abfliessens erlebt haben. Wir haben nus dann immer so geholfen, dass wir den abgeklemmten Schlanch ans dem Spülgefäss heransuahmen, einen Trichter anf den Schlanch setzten nnd nnn solange Salicylsänrelösnng anfgossen nnd wieder ablanfen liessen, bis die Passage frei war. Noch einfacher aber wird dieser Umstand zn beseitigen sein mittels des Verfahrens von A. Fraenk e ${ }^{1}$ ), der anstatt des einfachen Glasrohres ein gabelförmiges Dreiwegestück einschaltet, nnd nnn von dem einen Schenkel ans die Gerinnsel ans dem Heberrohr heransspült. Das Anftreten von grossen Fibrinmassen in dem Plenraranm, die das ganze Verfahren illnsorisch machen sollen, dürfte denn doch nicht so hänfig sein, wie Gläser es annimmt.

Wenn nus bei grösserer Unrnhe nnd Umherwerfen des Patienten die Gefahr zn drohen schien, dass bei nngenügender Anfsicht des Nachts die Heberdrainage zerstört würde, so haben wir einfach den Katheter mit einem Qnetschhahn abgeklemmt nnd ihn dann mit einer Binde an dem Thorax befestigt. Ebenso verfnhren wir, wenn die Eitersecretion in der Plenrahöhle schon beträchtlich abgenommen nnd wenn wir es für angezeigt hielten, den Patienten am Tage einige Stnnden ausserhalb des Bettes verbringen zn lassen.

Was nnn die Resnltate in nnseren Fällen anlangt, so möchte ich znnächst betonen, dass in nnseren 4 ersten Fällen das vollkommenste Resnltat erreicht worden ist, das man überhanpt von einer Heilmethode verlangen darf, nämlich eine völlige oder doch wenigstens nahezn völlige Restitntio ad integrnm der anatomischen nnd physiologischen Verhältnisse, nnd zwar hier der Brnstorgane; ja in nnserem Falle II nnd IV darf man es als wahrscheinlich bezeichnen, dass die Bülan'sche Methode mehr geleistet hat, als die Rippenresection hätte leisten können.

In nnserem zweiten Falle nämlich bestand das Empyem sicherlich 3-4 Monate, nnd in solchen Fällen kommt es erfahrnngsgemäss nach der Rippenresection zn grösseren Schrnmpfnngen des Thorax oder anch zn Fistelbildnngen, weil eben die Lnnge ohne die sangende Kraft der Heberdrainage sich nicht mehr vóllig ansdehnt, wie dies ja B̈̈lan in einem Falle seiner letzten Pnblication 2) klar gezeigt hat.

In unserem vierten Falle hatte der consnltirte Chirnrge, Herr Professor Witzel, wegen der vorhandenen Albnminnrie, wegen der Oedeme nud der hochgradigen Schwäche der Patientin die Ansführung der Rippenresection abgelehnt, nnd die von uns mit nnr geringem Mnthe unternommene Operation führte in 36 Tagen zn einem glänzend zn nennenden Resnltate.

Weiter zeigen nnsere geheilten Fälle, dass die Heberdrainage vor den janchigen Empyemen als einem "Noli me tangere屯 nicht znrückznschrecken brancht; nnsere beiden Fälle sind glatt geheilt, nnd damit ist die Ansicht von Leyden bestätigt, die übrigens anch Trendelenburg schon seit längerer Zeit seinen Schülern vorgetragen, dass nämlich die janchigen Empyeme nicht eine schlimmere Prognose geben, als die übrigen.

$\mathrm{Ob}$ nnn in nnseren beiden letzten Fällen, die mit dem Tode der.Patienten endeten, es möglich gewesen wäre, dnrch die Rippenresection den schlimmen Ausgang zn verhüten, das darf man wohl ziemlich sicher verneinen.
In dem einen Falle hatten wir es zn thnn mit einem seit zwei Monaten bestehenden Pyopnenmothorax tnbercnlosns; der Patient war änsserst elend nnd dyspnoisch nnd znr Vornahme einer Radicaloperation wenig geeignet; nachdem er sich nun bei Anlegnng der Heberdrainage erholt, hätte es sicher der ansgedehnten Schedeschen Thoraxresection bedurft, nm eine Ausheilnng der Lnngenfistel zn erzwingen, nnd da dieses Verfahren eine Behandlnngsdaner meist von mindestens einem Jahr in Ansprnch ninumt, so war bei dem geringen Kräfteznstand des Patienten diese Heilmethode wohl kanm mit Erfolg durchznführen.

In nnserem $Z$ weiten tödtlich geendeten Falle handelte es sich nm einen Patienten, der dnrch die seit längerer Zeit stattfindende Resorption von pntriden. Stoffen hochgradig inficirt war; anch die Resection hätte hier den Patienten nicht mehr retten können, weil eine rasche nnd gründliche Ansränmnng der zerfetzten Plenrahöhle, von der ans die janchige Infiltration zwischen Plenra costalis nnd Hantzellgewebe fortgeschritten war, wohl kanm gelnngen wäre. Ueberdies war durch die Bülan'sche Drainage alles ansgspüllt worden, was zn entleeren war.

Ueber die Heilnngsdaner in nuseren 4 geheilten Fällen will ich hier nur noch knrz bemerken, dass sie im Mittel 55 Tage betrng, nnd ich möchte dies nm so mehr hervorheben, als ja König in seiner letzten Mittheilnng ${ }^{1}$ ) besonders betont, dass $71 \%$ seiner Fälle innerhalb 2 Monaten geheilt waren.

Was nnn die Indication für das B ̈̈lan'sche Verfahren anlangt, so glanbe ich, dass man berechtigt ist, dasselbe in allen Fällen von einfachen Empyemen, die janchigen nnd tnbercnlösen nicht ansgeschlossen, anznwenden, nnd zwar einmal, weil das Verfahren, wie die Resnltate von Bülan, Simmonds, Curschmann, Wölfler, Leyden n. a. beweisen, in sehr vielen Fällen gnte Erfolge hat, nnd dann weiter, weil es das einfachere, weniger eingreifende Verfahren ist.

Anf letzteren Pnnkt mnss man doch jedenfalls besonders achten, wenn man einem Patienten, besonders einem sehr elenden, ein Operationsverfahren vorschlägt, ganz abgesehen davon, dass die meisten messerschenen Patienten sich e $\mathrm{h}$ er zn einer solchen Operation werden bestimmen lassen. Man nmgeht dabei die Gefahren der Chloroformnarkose, die doch sicherlich vorhanden sind, wie dies ja einmal die mitgetheilten Todesfälle während der Narkose beweisen, und die anch von operationslnstigen Chirnrgen derart geschent werden, dass sie die Rippenresection eventnell ohne Narkose vornehmen. ${ }^{2}$ ) Ferner findet man nach der B̈̈lan'schen Behandlnng des Empyemes keine grössere Verändernng des Thorax, während nach der Rippenresection öfters, besonders bei Erwachsenen, eine Einsenknng der betreffenden Thoraxhälfte stattfindet, die dann wieder eine grössere Lnngenschrnmpfung zur Folge haben muss.

Von den Chirnrgen wird dem Bülan'schen Verfahren die Berechtignng meist nnr dann zngesprochen, wenn sie sich in Verlegenheit befinden, d. h. wenn sie es mit einem doppelseitigen Empyem zn thnn haben, oder wenn der Patient zn elend vor der Operation ist, nm Zeit zn gewinnen. Wenn aber in solch' verzweifelten Fällen dem Verfahren die Berechtignng znerkannt wird, so ist es nm so weniger zn begreifen, warnm man ihm in einfacheren Fällen dieselbe versagen will. Es ist hente die Behanptnng der Gegner nicht mehr gerechtfertigt, dass man immer das sicherste Mittel, i. e. die Rippenresection, znerst in Anwendnng bringen müsse, seitdem die Statistiken von Curschmann, Immermann, Wölfler existiren, die sich mit denen der Chirnrgen, nnd zwar anch der nenesten von $K o ̈ n i g$ in Bezng anf die gnten Erfolge rnhig messen können, wenn sie anch allerdings in der Zahl der Fälle dieselben noch nicht erreichen.

Selbst wenn anch die Heberdrainage einmal versagen sollte, bei mehreren abgesackten Eiterherden in der Plenrahöhle oder bei massenhaften Fibrinansammlnngen, so kann man immer noch die Rippenresection anschliessen, nachdem man den Patienten znerst 8--14 Tage mit der Heberdrainage behandelt hat, ohne damit dem Patienten einen grösseren Nachtheil gebracht zn haben.

Wenn die Chirnrgen, nm noch bessere Resnltate als bisher dnrch die Rippenresection zn erhalten, immer wieder verlangen, dass ihnen möglichst früh die Empyeme znr Operation zngeschickt werden, so müssen anch wir den Aerzten gegenüber dringend diesen Wnnsch anssprechen, weil bei diesen einfachen frischen Fällen anch für die Heberdrainage die Bedingnngen für einen raschen nnd völligen $\mathbf{E r}-$ folg am günstigsten sind; möglichst früh soll sich der Arzt durch die Probepnnction darüber Gewissheit verschaffen, welcher Natnr das Exsndat im Plenraranme ist, nnd wenn es ein eitriges ist, dann sofort znr Drainage schreiten. Ich kann aber allerdings nicht I mm erman n zustimmen, dass es erlanbt ist, bei constatirtem Empyem znr Feststellnng der bacteriologischen Species, die die Eiternng veranlasst hat, beliebige Probepnnctionen zu machen; denn die Mög-

1) Siehe diese Wochenschrift 1891 No. 17, p. 597.

2) Zeitschrift für klin. Medicin Band 18 p. 34 .

11) Berl. klin. Wochenschrift 1891 No. 10

3. König l. c. 
lichkeit, an der Punctionsöffnung eine Infection hervorzurufen, ist doch immer gegeben, wie das auch die Phlegmonen, die nach Punction von tuberculősen Empyemen auftreten, beweisen, und wie das ja auch die in unserem vierten Falle von der Probepunctionsoffnung sich ausbreitende Phlegmone beweist.

$\mathrm{Ob}$ bei Pyopneumothorax mit offener innerer Lungenfistel die Heberdrainage anzuwenden ist, darüber müssen noch weitere Erfahrungen gesammelt werden; bei dem tuberculösen Pyopneumothorax scheint man jedoch im allgemeinen die symptomatische Behandlung durch wiederholte Punctionen zu bevorzugen, da bisher auch die Rippenresection in diesen Fällen sehr wenig befriedigende Resultate geliefert hat. 